\title{
Mantle-driven geodynamo features—effects of post-Perovskite phase transition
}

\author{
Hagay Amit and Gaël Choblet \\ CNRS, Université de Nantes, Nantes Atlantique Universités, UMR CNRS 6112, Laboratoire de Planétologie et de Géodynamique, \\ 2 rue de la Houssinière, F-44000 Nantes, France
}

(Received January 19, 2009; Revised June 19, 2009; Accepted August 6, 2009; Online published December 21, 2009)

\begin{abstract}
Exploring the impact of the heterogeneous lower mantle on the geodynamo requires knowledge of the heat flux anomaly across the core-mantle boundary. Most studies so far used a purely thermal interpretation of seismic shear wave anomalies to assign heterogeneous heat flux boundary conditions on numerical dynamo models, ignoring phase transition or compositional origins. A recent study of mantle convection (Nakagawa and Tackley, 2008) provides guidelines to include such non-thermal effects. Here we construct maps of heat flux across the core-mantle boundary based on a lower mantle tomography model (Masters et al., 2000) with a combined thermal and post-Perovskite phase transition interpretation. We impose these patterns as outer boundary conditions on numerical dynamo simulations and study the impact of accounting for post-Perovskite effects on the long-term time-average properties of the dynamo. We then compare our results with geophysical observations. We find in all cases that surface downwellings associated with cyclones concentrate intense non-axisymmetric magnetic flux at high-latitudes, the surface flow contains a large anticlockwise vortex at mid-latitudes of the southern hemisphere, and the inner boundary buoyancy flux is dominated by a $Y_{2}^{0}$ pattern. Boundary-driven time-average surface flow with some equatorial asymmetry is organized in the shell by quasi-axial convective rolls that extract more buoyancy from low-latitudes of the inner-boundary. These positive inner boundary buoyancy flux structures are found at low-latitudes of the northern hemisphere, in some places due to cyclonic flow at mid-latitudes of the southern hemisphere connecting with higher latitude cyclonic flow in the northern hemisphere. Accounting for post-Perovskite effects improves the recovery of several geodynamo observations, including the Atlantic/Pacific hemispherical dichotomy in core flow activity, the single intense paleomagnetic field structure in the southern hemisphere, and possibly the $m=1$ dominant mode of inner-core seismic heterogeneity.
\end{abstract}

Key words: Geodynamo, mantle tomography, post-Perovskite, geomagnetic field, core flow, inner core.

\section{Introduction}

The impact of the heterogeneous lower mantle on the geodynamo has been the focus of many studies in recent years (Glatzmaier et al., 1999; Bloxham, 2002; Olson and Christensen, 2002; Christensen and Olson, 2003; Aubert et al., 2007, 2008; Gubbins et al., 2007; Willis et al., 2007). These studies attempted to explain the existence of non-axisymmetric features on various time-scales in Earth's core using numerical dynamos with heterogeneous heat flux boundary conditions based on lower mantle tomographic models (Su et al., 1994; Masters et al., 1996, 2000) and a thermal core-mantle coupling assumption (Bloxham and Gubbins, 1987). Comparisons of the dynamo models' output with the time-average historical and paleomagnetic field (Bloxham, 2002; Olson and Christensen, 2002; Gubbins et al., 2007) and with persistent features in inverted core flows from geomagnetic secular variation (Olson and Christensen, 2002) showed some encouraging similarities. In a recent study, Aubert et al. (2008) reconciled three observed non-axisymmetric geodynamo features: The timeaverage paleomagnetic field (Kelly and Gubbins, 1997), the time-average core flow inferred from the historical geomag-

Copyright (c) The Society of Geomagnetism and Earth, Planetary and Space Sciences (SGEPSS); The Seismological Society of Japan; The Volcanological Society of Japan; The Geodetic Society of Japan; The Japanese Society for Planetary Sciences; TERRAPUB. netic secular variation (Amit and Olson, 2006), and the eastwest hemispherical dichotomy in the seismic properties of the upper inner-core (Tanaka and Hamaguchi, 1997).

Amit et al. (2008) took a different approach. They inferred the radial shear of the mantle-driven flow from numerical dynamos and the thermal distribution at the top of the core from mantle tomography to construct a simple thermal wind model driven by mantle heterogeneity. Comparison of their thermal wind model with the time-average core flow model of Amit and Olson (2006) shows good recovery of the anticlockwise vortices below the southern Atlantic and Asia, the latter associated with the east-west inner-core dichotomy (Aubert et al., 2008). However, the thermal wind model is in disagreement with other steady flow features, especially in the western hemisphere.

Despite significant regions where the models' timeaverage magnetic field, flow and inner-core boundary buoyancy flux seem to agree with the observations, other regions still show discrepancies. The possible reasons for the discrepancies may be divided into three classes:

- Numerical models-Numerical dynamos provide a self-consistent solution to the full set of magnetohydrodynamics equations, but their output varies depending on the parameters chosen, and due to computational limitations they do not operate in Earth-like parametric regime. The thermal wind model of Amit 
et al. (2008) relies on simplifying assumptions.

- Geodynamo observations-First, paleomagnetic field models suffer from poor data coverage and quality which might pose difficulty on detecting nonzonal features (Carlut and Courtillot, 1998), although resemblances among paleomagnetic field models obtained using various datasets and methods suggest that these non-axisymmetric features are robust (Constable, 2007). Second, the time-average core flow from the inversions of the geomagnetic secular variation spans only 150 years and therefore might contain transients, although Aubert et al. (2007) argued that a significant correlation between the 150-years and the long-term time-averages is expected. Other uncertainties associated with core flow inversions might also bias these models (for a recent review see Holme, 2007). Finally, the incomplete seismic data coverage of the upper inner-core, especially at high-latitudes (Niu and Wen, 2001), prevents detailed detection of inner-core heterogeneities.

- Lower mantle thermal anomalies-A thermal interpretation of mantle tomography models is incomplete, as evidenced by the anti-correlation between seismic shear and bulk sound velocities in the lower mantle ( $\mathrm{Su}$ and Dziewonski, 1997; Masters et al., 2000). Studies of the core-mantle boundary region suggest that compositional (Deschamps et al., 2007; Ritsema et al., 2007), post-Perovskite phase transition (Murakami et al., 2004; Oganov and Ono, 2004) and partial melting (Williams et al., 1996; Lay et al., 2004) origins should be considered as well.

This paper addresses the last of these three points. So far most studies of mantle control on core dynamics assumed a linear relationship between the patterns of the heat flux anomaly across the core-mantle boundary $\delta q$ and the lower mantle seismic shear velocity anomaly $\delta v_{s}$ ( $S$-wave core reflections and diffractions have larger amplitudes than their $P$-wave counterparts and thus resolve better the lowermost mantle, see Thurber and Ritsema, 2007). In a recent compelling paper, Nakagawa and Tackley (2008) used 3D mantle convection models to study the relation between $\delta q$ and $\delta v_{s}$ due to thermal, compositional and phase transition anomalies in the lower mantle. Here we apply their results to the mantle tomography model of Masters et al. (2000) in order to construct heat flux distributions that incorporate non-thermal effects.

Seismic evidence of dense partial melts at Ultra-Low Velocity Zones (ULVZ) has been reported for the base of the mantle (Williams et al., 1996; Lay et al., 2004), consistent with both melting temperatures of deep mantle phases and the thermal history of the Earth (Labrosse et al., 2007). Partial melting, if present, would modify the $\delta q$ to $\delta v_{s}$ relation in the hottest regions: While seismic velocities will be reduced, the possibly dramatic decrease of the bulk viscosity associated with even small amounts of melt will enhance heat transfer (Matsumoto et al., 2006). As a consequence, the $\delta q$ vs. $\delta v_{s}$ curve would become flat for the smallest $\delta v_{s}$. Note however, that if important, this phenomenon is probably restricted to localized areas, namely the ULVZ regions (maybe coinciding with the roots of hot mantle plumes, see Williams and Garnero, 1998). In addition, parameterization of the influence of melting on the $\delta q-\delta v_{s}$ relationship is still lacking, contrary to the effects of post-Perovskite and compositional heterogeneities that were modeled by Nakagawa and Tackley (2008). For both reasons, the effects of partial melting are not included in the present study.

Numerical simulations (Tackley, 2002) and laboratory experiments of two-layer thermal convection in miscible viscous fluids (Le Bars and Davaille, 2004) show that the thermo-chemical dynamics of the lower mantle is mostly controlled by the buoyancy number measuring the ratio between density variations caused by composition to those caused by temperature. As shown by Nakagawa and Tackley (2008), neither very small nor very large buoyancy numbers lead to significant deviations from the linear $\delta q$ to $\delta v_{s}$ relation. If the density variation associated with a thin layer of denser material initially located above the core-mantle boundary is too small, the initial layering is quickly swept away by thermal convection. On the other hand, a large density variation results in stable stratification and once again an isochemical $\delta q$ to $\delta v_{s}$ relation. In an intermediate buoyancy number scenario, the dense material is swept into ridge-shaped piles where enhanced internal heating causes a small heat flux. In this configuration, the largest $\delta v_{s}$ is still associated with the largest $\delta q$, but the smallest $\delta v_{s}$ does not coincide with the smallest $\delta q$ (below the dense piles where $\delta v_{s}$ is larger due to composition) but with the edges of the piles where $\delta q$ is moderate, resulting in complex non-unique $\delta v_{s}$ to $\delta q$ mapping (Nakagawa and Tackley, 2008). Moreover, while regional tomographic studies have highlighted sharp features (Ni et al., 2002; To et al., 2005) that could be associated with ridge-like seismic structures of compositional origin, such features are not resolved in global tomography and geomagnetic field models.

In contrast, post-Perovskite effects can be accounted for in a rather simple way, since the relationship between $\delta q$ and $\delta v_{s}$ when both thermal and phase transition effects are considered is unique (Nakagawa and Tackley, 2008). In this paper we therefore focus on these isochemical cases. We construct maps of $\delta q$ based on a non-linear relation to the tomographic model of $\delta v_{s}$, representing both thermal and phase transition effects. Those maps are imposed as heterogeneous boundary conditions on numerical dynamo simulations. The time-average properties of the dynamo models are compared with relevant observations, and the geophysical implications are discussed.

\section{Method}

We use 3D self-consistent numerical dynamos in a spherical shell. For details on the method and governing equations see Aubert et al. (2007). We implement the simulation MAGIC (Wicht, 2002), originally coded by Gary Glatzmaier. Because we wish to focus on the effects of various heat flux boundary patterns, we do not change the control parameters. For all cases we use a heat flux based Rayleigh number $R a \equiv \alpha g_{0} q_{0} D^{4} / k \kappa v=10^{6}$ (which is about five times the critical value for the onset of nonmagnetic convection), an Ekman number $E \equiv v / \Omega D^{2}=$ $3 \cdot 10^{-4}$, a Prandtl number $\operatorname{Pr} \equiv \nu / \kappa=1$, a magnetic 
Prandtl number $P m \equiv v / \lambda=3$ and a heat flux anomaly amplitude $q^{*} \equiv\left(q_{\max }-q_{\min }\right) / 2 q_{0}=0.5$, where $\alpha$ is thermal expansion coefficient, $g_{0}$ gravity on the outer boundary, $q_{0}$ mean heat flux across the outer boundary, $D$ shell thickness, $k$ thermal conductivity, $\kappa$ thermal diffusivity, $v$ kinematic viscosity, $\Omega$ rotation rate and $\lambda$ magnetic diffusivity. The inner to outer radii ratio is 0.35 . The boundary conditions are rigid for the velocity and insulating for the magnetic field. The inner boundary has fixed buoyancy, and the outer boundary has a prescribed heat flux pattern. The grid includes 41 radial, 192 longitudinal and 96 latitudinal points, and in spectral space the fields were expanded up to degree and order 64.

Based on the kinetic and magnetic energies in the entire shell, the magnetic Reynolds number (describing the ratio of magnetic advection to magnetic diffusion) in our simulations is $R m=125$, and the Elsasser number (describing the ratio of Lorentz to Coriolis forces) is $\Lambda=0.93$. The instantaneous surface magnetic fields have some morphological features that resemble the geomagnetic field, most notably the dominance of the axial dipole and high-latitude intense normal magnetic flux patches, but the field does not reverse. The instantaneous flow is dominated by strongly time-dependent nearly axial convective columns.

For proper time-averaging, the various time-scales of the system must be exceeded. These include the vortex turnover time $\tau_{u}=D / U$ (where $U$ is the rms flow in the shell), the viscous diffusion time $\tau_{v}=D^{2} / \nu$, the thermal diffusion time $\tau_{\kappa}=D^{2} / \kappa$ and the magnetic diffusion time $\tau_{\lambda}=D^{2} / \lambda$. Each simulation was ran for about 2.5 magnetic diffusion times, corresponding to 7.5 viscous and thermal diffusion times, and 312.5 vortex turnover times, comparable to the length of the longer simulations of Aubert et al. (2007). We also extended one simulation in 1.25 magnetic diffusion times and verified that the time-average fields are practically unchanged. All steady fields reported here correspond to averaging over the entire simulation time of $2.5 \tau_{\lambda}$.

Various heterogeneous heat flux patterns were imposed on the outer boundary. As in previous studies, the reference purely thermal interpretation $\delta q_{T}$ (i.e. with no phase transition) is linear to the lowermost mantle seismic shear velocity $\delta v_{s}$ tomography model of Masters et al. (2000):

$$
\frac{\delta q_{T}}{q_{T}}=C \frac{\delta v_{s}}{v_{s}}
$$

where $C$ is a non-dimensional constant that relates the amplitude of the shear velocity anomaly with the amplitude of the heat flux anomaly. In practice, the input parameter $q^{*}$ determines the magnitude of the imposed heat flux heterogeneity. Note that we neglect the curvature found by Nakagawa and Tackley (2008) describing their purely thermal case; this very mild non-linearity (due to perhaps transient or internal heating effects) is secondary in the context of the present study. The other heat flux patterns are based on the results of Nakagawa and Tackley (2008) for the isochemical cases in which post-Perovskite phase transitions were accounted for. They found that the nearly perfectly linear $\delta q$ to $\delta v_{s}$ relation in the purely thermal cases transforms to an inclined step function when post-Perovskite phase transitions are included. This shape is related to the assumption that post-Perovskite is a few \% seismically faster than Perovskite and at these depths the phase transition occurs at a specific temperature. The spreading of the effect is well-approximated by a tanh function. Following equation (B8) of Nakagawa and Tackley (2008), we formulate the thermal-phase interpretation $\delta q_{T p}$ as follows:

$$
\frac{\delta q_{T p}}{q_{T p}}=\frac{\delta q_{T}}{q_{T}}+A\left[0.5\left(\tanh \left(-\frac{1}{B} \frac{\delta q_{T}}{q_{T}}\right)-1\right)\right]+D
$$

The non-dimensional parameters in (2) are the amplitude $A$, the tanh spreading factor $B$, and the offset $D$.

Nakagawa and Tackley (2008) used an increase of $A=$ $2 \%$ of seismic shear velocity associated with the transition from Perovskite to post-Perovskite. This value globally reflects the results of first principle calculations (e.g. Stackhouse et al., 2006). Wentzcovitch et al. (2006) suggested that $A$ ranges between $0 \%-3 \%$ due to uncertainties, with a preferred value of $2 \%$. While there is a general agreement that the phase transition effect is minor on $P$-waves velocity, large discontinuities were reported for $S$-waves, from 1\% (Iitaka et al., 2004) to 4\% (Wookey et al., 2005; Lay et al., 2006). Laboratory measurements at appropriate pressures (but at room temperatures) tend to favor a moderate phase transition effect of $A=0.5 \%$ (Murakami et al., 2007 ), indicating that anisotropy could be required to explain the observed seismic discontinuities at the $\mathrm{D}^{\prime \prime}$ layer. As noted by Nakagawa and Tackley (2008), if such a large effect of anisotropy was present, it would further complicate the relationship between the heat flux and the seismic shear velocity. Low $A$ values could also be caused by the presence of iron or aluminium (Tsuchiya and Tsuchiya, 2006).

We therefore examine $A=2 \%$ and $3 \%$ to test the dependency of our results on the amplitude. Smaller amplitudes are bounded between these values and the purely thermal case $(A=0)$. The spreading factor $B$ was fitted numerically by Nakagawa and Tackley (2008); We treat $B$ as a free parameter. We note that for a given amplitude $A$, the spreading factor $B$ is constrained by reaching the tanh asymptote on the one hand, and maintaining an inclined step function (rather than $Z$-like curve) on the other hand. The offset $D$ is set so that $\int_{S} \delta q_{T p} d S=0$ where $S$ is the surface of the outer boundary. Finally, we truncate the resulting heat flux patterns at spherical harmonic degree 9 (as in Aubert et al., 2007) for consistency with the spatial resolution that we use for $\delta q_{T}$.

Figure 1 shows four $\delta q$ vs. $\delta v_{s}$ models. The black curve represents a purely thermal linear interpretation of seismic shear velocity $\delta q_{T}$ (1). The other three curves exhibit nonlinear tanh-dependency $\delta q_{T p}$ prescribed by (2) with different $A$ and $B$ values to account for post-Perovskite phase transition effects. Figure 2 displays the core-mantle boundary heat flux anomalies maps corresponding to these scatter plots. Although the patterns are very similar, the relative magnitudes of the positive/negative heat flux anomalies structures varies. When the post-Perovskite effect is considered, the positive structures weaken and spread over larger areas, whereas the negative structures remain strong and more localized (Fig. 2(b)). This is due to the asymmetric 


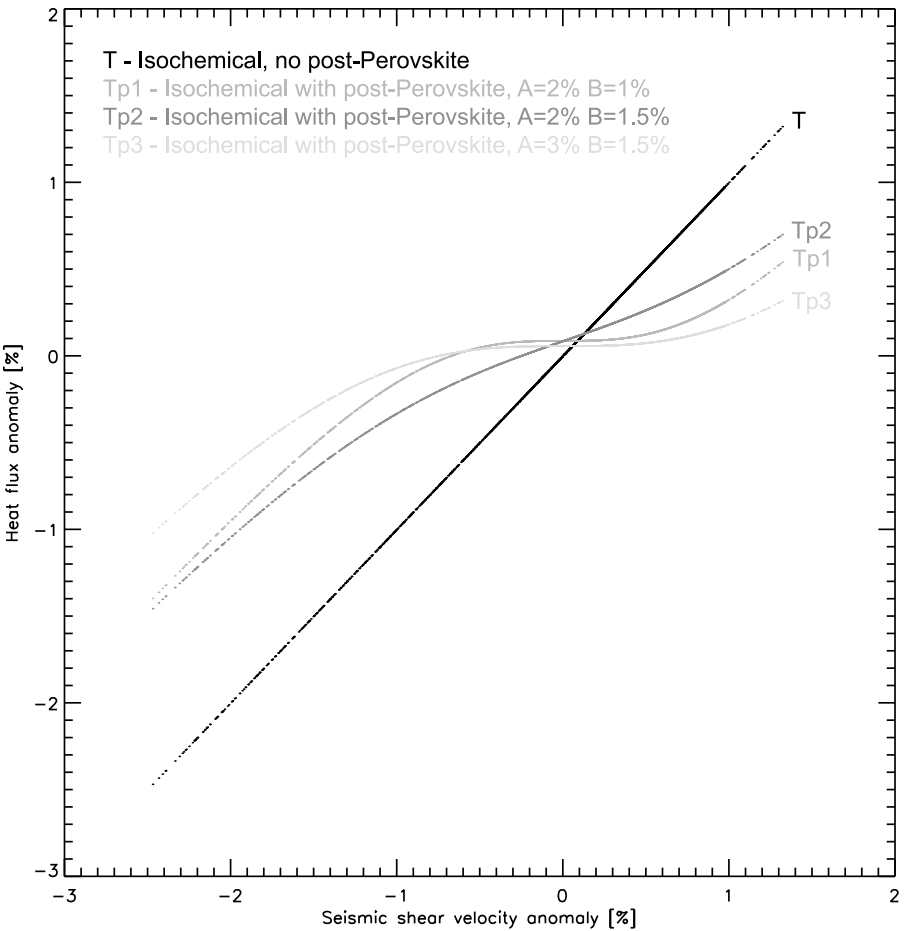

Fig. 1. Scatter plots of heat flux vs. seismic shear velocity anomalies based on the tomographic model of Masters et al. (2000). Black curve is the conventional linear interpretation based on Eq. (1), grey curves are based on Eq. (2).

(a) $\mathrm{T}$

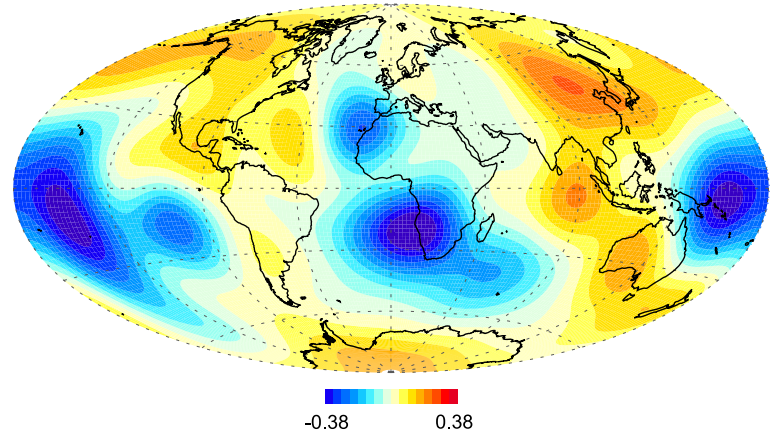

(c) Tp2

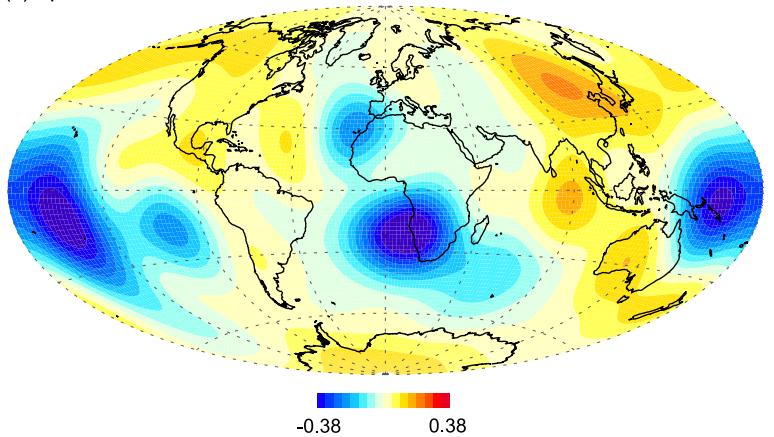

(b) Tp1

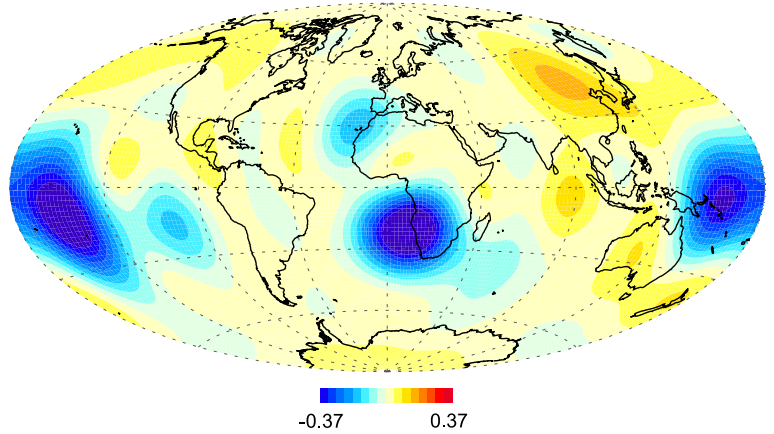

(d) $\mathrm{Tp} 3$

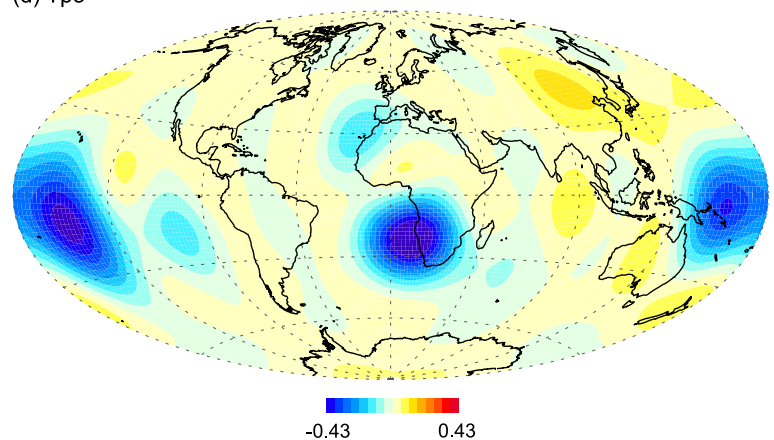

Fig. 2. Core-mantle boundary heat flux anomalies normalized by the mean heat flux $\delta q / q_{0}$ based on the mantle tomography model (Masters $e t$ al., 2000). A thermal linear interpretation T (a), and thermal-phase non-linear models Tp1 $(A=2 \%$ and $B=1 \%, \mathrm{~b}), \mathrm{Tp} 2(A=2 \%$ and $B=1.5 \%, \mathrm{c})$, and $\operatorname{Tp} 3(A=3 \%$ and $B=1.5 \%, \mathrm{~d})$.

distribution of the tomographic model that contains larger amplitude narrower regions of negative seismic velocity anomalies $\left(\delta v_{s}, \delta q_{T}<0\right)$ as opposed to broader regions of weaker positive anomalies (Fig. 2(a)). The tanh function merely shifts the large negative $\delta v_{s}$ with little change in their relative sizes, whereas it strongly reduces intermediate $\delta v_{s}$ while weakly reduces very low $\delta v_{s}$ (Fig. 1), resulting in the spreading effect of the positive $\delta q_{T}$. The maps in Fig. 2 were imposed as outer boundary heat flux patterns on the numerical dynamos. 
(a) $\mathrm{T}$

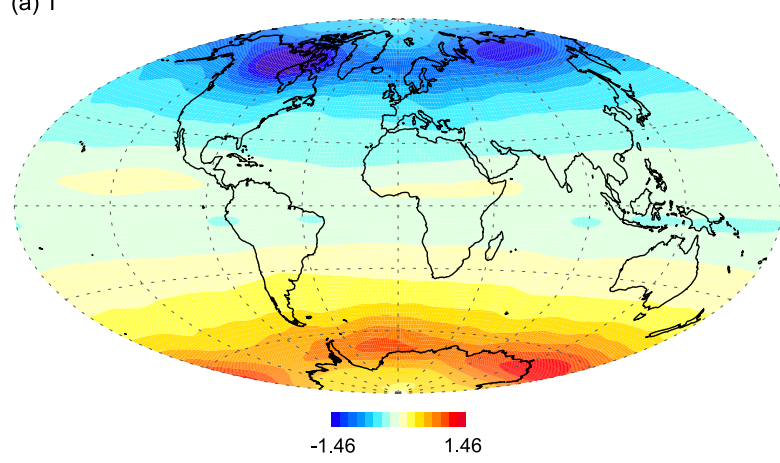

(c) Tp2

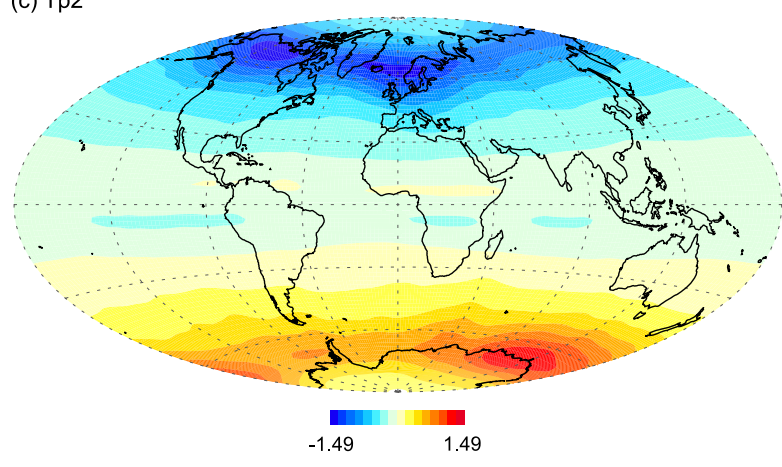

(e) KG97

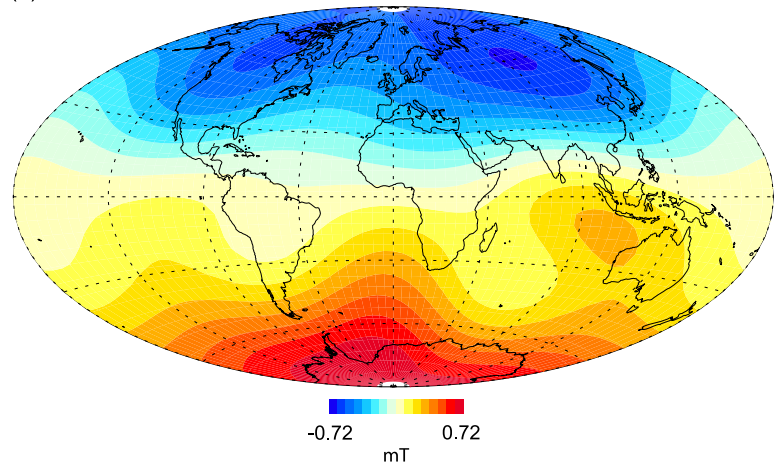

(b) $\mathrm{Tp} 1$

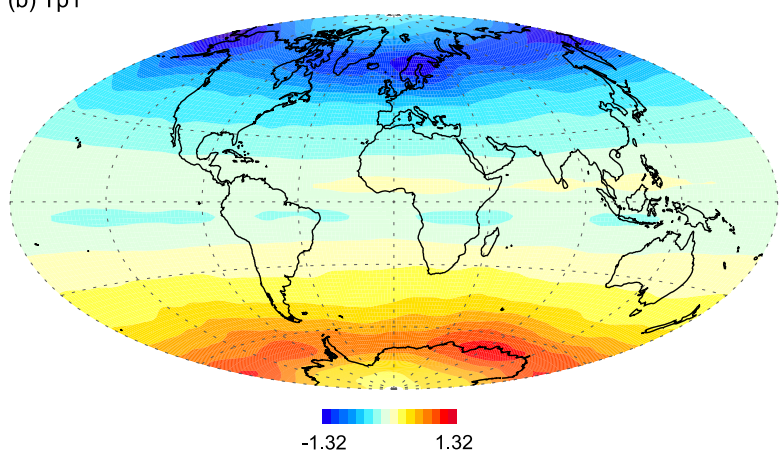

(d) $\mathrm{Tp} 3$

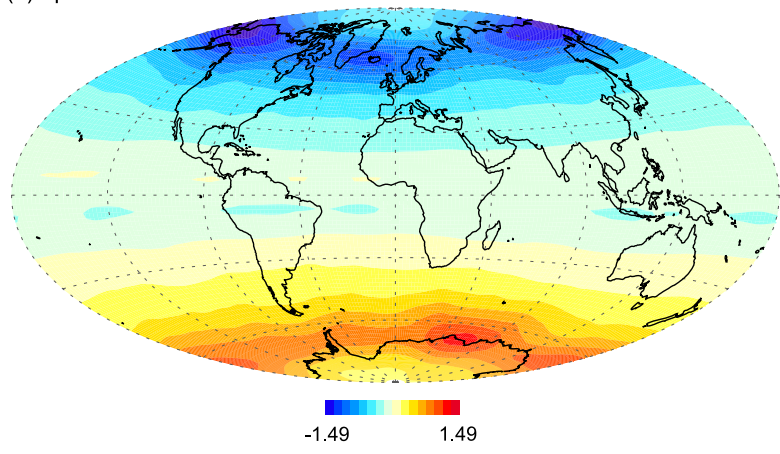

(f) GUFM1

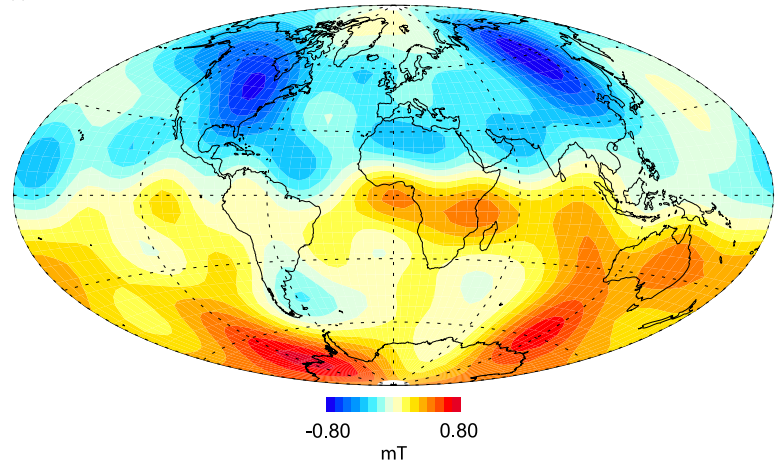

Fig. 3. Time-average radial magnetic field on the core-mantle boundary: (a) Model T; (b) Model Tp1; (c) Model Tp2; (d) Model Tp3; (e) Time-average paleomagnetic field model over the past 5 million years from Kelly and Gubbins (1997); (f) Time-average geomagnetic field model between 1840-1990 from Jackson et al. (2000). The dynamo models' magnetic fields are given in units of $\sqrt{\rho \mu_{0} \lambda \Omega}$ where $\rho$ is the fluid density and $\mu_{0}$ permeability of free space, the observed fields are given in $\mathrm{mT}$.

\section{Results}

Because of the time disparity between the mantle and the outer core, mantle control is best seen on long-term timeaverages (Bloxham, 2002; Olson and Christensen, 2002; Christensen and Olson, 2003; Aubert et al., 2008). We examine the time-average properties of our dynamo models with various imposed heat flux boundary conditions corresponding to various thermal and phase transition interpretations of the seismic tomography model. Motivated by the available geodynamo observations, we focus on three timeaverage dynamo properties: The radial magnetic field on the outer boundary, the flow at the top of the shell, and the buoyancy flux across the inner boundary.

Figure 3 shows the time-average radial magnetic field $B_{r}$ on the outer boundary for the purely thermal interpretation of the seismic data (3(a), from hereafter case T) and for cases with thermal-phase interpretations $(3(b-d)$, from hereafter cases $\mathrm{Tp} 1, \mathrm{Tp} 2$ and $\mathrm{Tp} 3$, respectively). The radial magnetic field in the northern hemisphere in case $\mathrm{T}$ (Fig. 3(a)) exhibits two intense non-dipolar structures below Canada and Siberia. In case Tp1 the structures are found more to the west below Alaska and Britain (Fig. 3(b)), in case Tp2 the structures appear below Canada and Britain, and in case Tp3 below Alaska and a weaker patch below Iceland. In the southern hemisphere, case $\mathrm{T}$ has two intense magnetic field features below the Atlantic and Indian Oceans, but only the latter appears in cases Tp1 and Tp2. Case Tp3 has an intense magnetic flux patch below the Indian Ocean, and another weaker patch below the western Pacific.

Figure 4 presents the time-average flow just below the Ekman boundary layer for all studied cases. The locations of $B_{r}$ structures correlate well with the locations of cyclones. For example, note in case $\mathrm{T}$ the three cyclones 
(a) $\mathrm{T}$

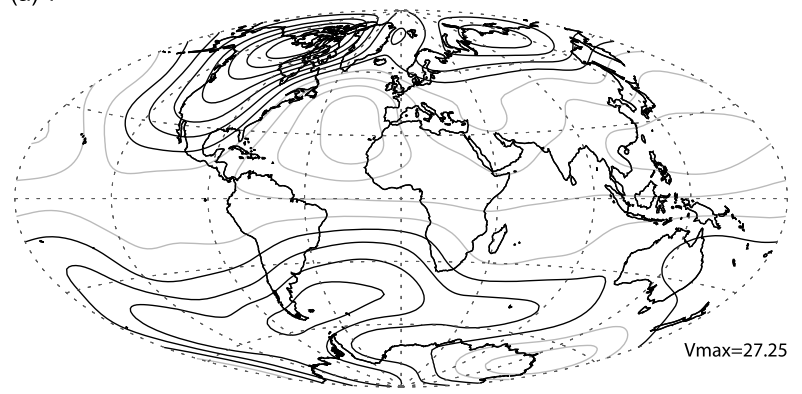

(c) Tp2

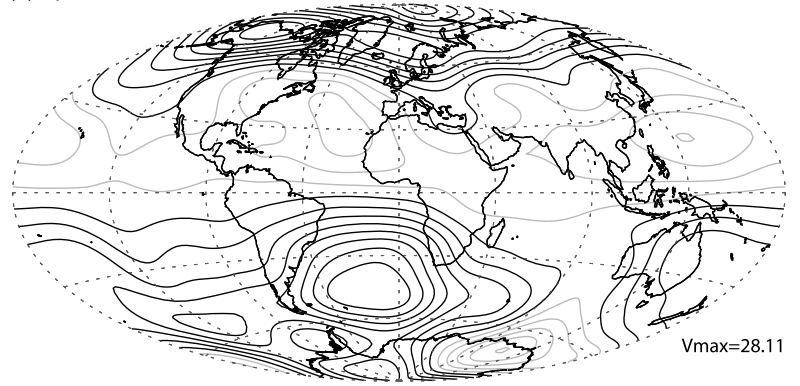

(e) AC08FF

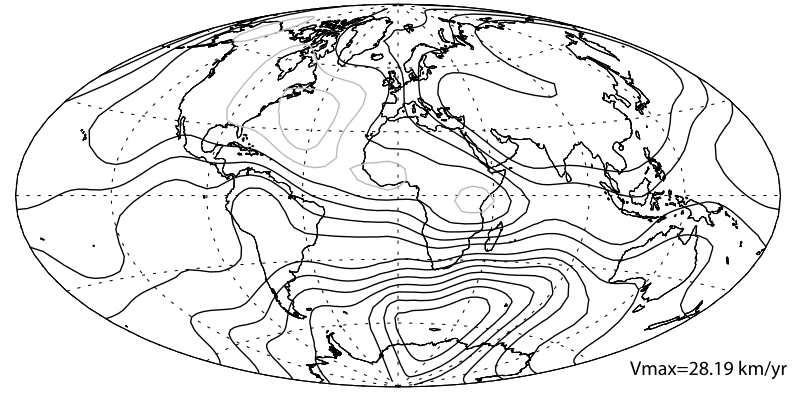

(b) $\mathrm{Tp} 1$

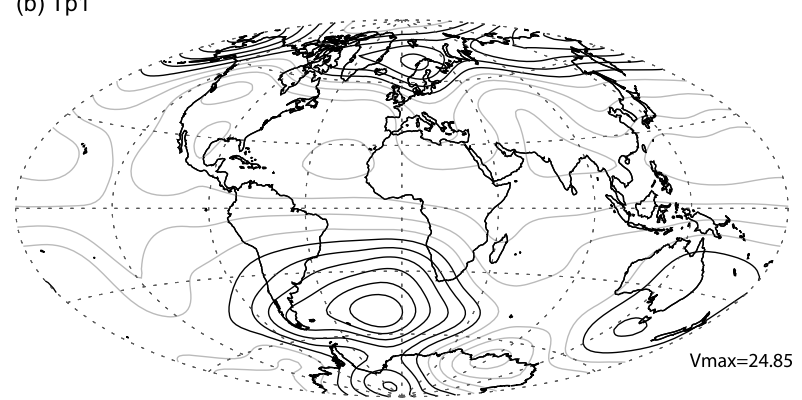

(d) $\mathrm{Tp} 3$

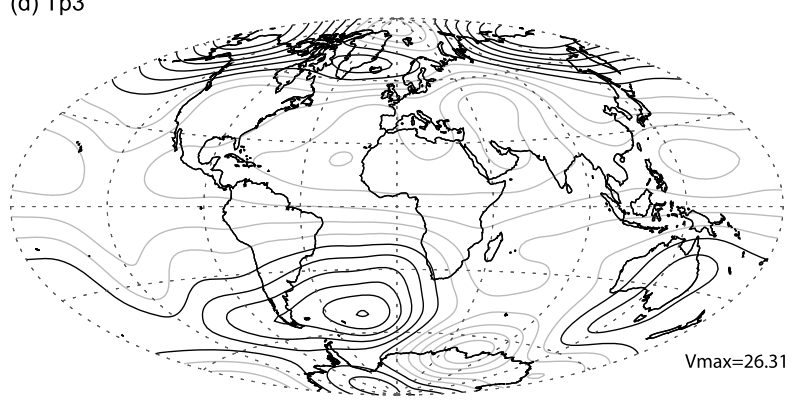

(f) AC08DIF

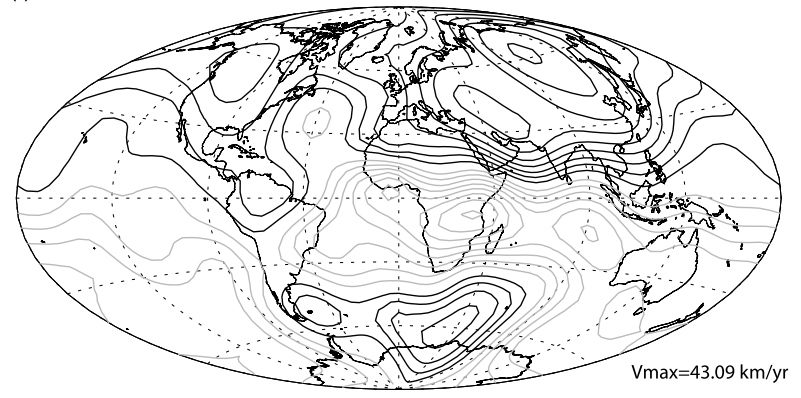

Fig. 4. Time-average toroidal flow at the top of the core: (a) Model T; (b) Model Tp1; (c) Model Tp2; (d) Model Tp3; (e) From the frozen-flux model of Amit and Christensen (2008); (f) From the diffusive model of Amit and Christensen (2008). Black/grey streamlines denote anticlockwise/clockwise circulation (contour intervals in e and $\mathrm{f}$ are the same). Maximum dynamo models' velocities are given in units of $v / D$, maximum inverted core flows are given in $\mathrm{km} / \mathrm{year}$.

below Canada, Siberia and longitude $135^{\circ} \mathrm{E}$ in Antarctica (Fig. 4(a)). The same longitudinal shift of $B_{r}$ structures among the cases (Fig. 3) can be observed for the flow structures as well. Another important flow feature in case $\mathrm{T}$ is a large anticlockwise vortex centered below the southern Atlantic encompassing most of the southern hemisphere with broad westward flow at mid-latitudes. A similar vortex appears in the other cases, but in cases Tp1 (Fig. 4(b)) and Tp3 (Fig. 4(d)) it is more confined to the Atlantic hemisphere.

The patterns of time-average buoyancy flux anomalies across the inner boundary of the models are given in Fig. 5. The inner boundary buoyancy flux contains a dominant $Y_{2}^{0}$ signature in all cases, with positive anomalies at lowlatitudes and negative anomalies at high-latitudes. Note that in all cases the low-latitudes positive inner buoyancy flux anomalies are centered at the northern hemisphere. The longitudinal dependency of the positive low-latitude anomalies in cases $\mathrm{T}$ and $\mathrm{Tp} 2$ is characterized by two isolated struc- tures (Figs. 5(a) and (c)), with the latter structures shifted westward. In contrast, in cases Tp1 and Tp 3 the positive inner boundary buoyancy flux is more spread with peaks below east Africa (Fig. 5(b)) or eastern Pacific (Fig. 5(d)). For a more quantitative analysis, we averaged the positive part of the inner boundary buoyancy flux anomalies along longitude lines (Fig. 6), and computed the ratio of the two lowest azimuthal modes $R_{12}=\delta q(m=1) / \delta q(m=2)$. In case Tp 2 the dominance of the $m=2$ mode is clear in the two comparable structures as reflected by the low $R_{12}$ value. Two structures dominance also appears in case T, but the Central American one is much stronger, giving rise to a relatively larger $m=1$ and $R_{12}$. The more spread distributions in cases Tp1 and Tp3 are also evident in Fig. 6. The peak in case Tp1 is expressed by a much larger $R_{12}$, indicating an increased relative $m=1$ in this case.

The differences of surface radial magnetic field, flow at the top of the shell, and inner-core boundary buoyancy 
(a) $\mathrm{T}$

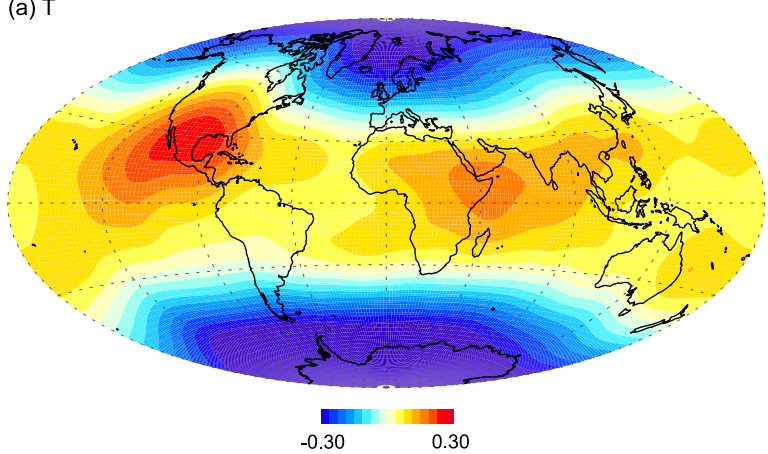

(c) Tp2

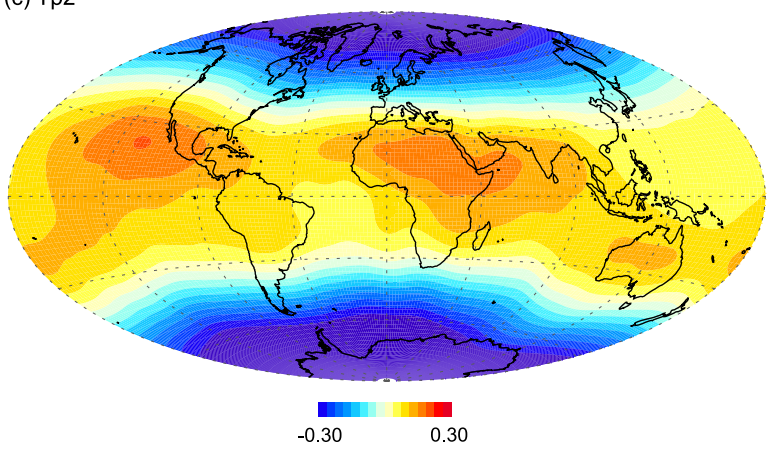

(e) YSK96

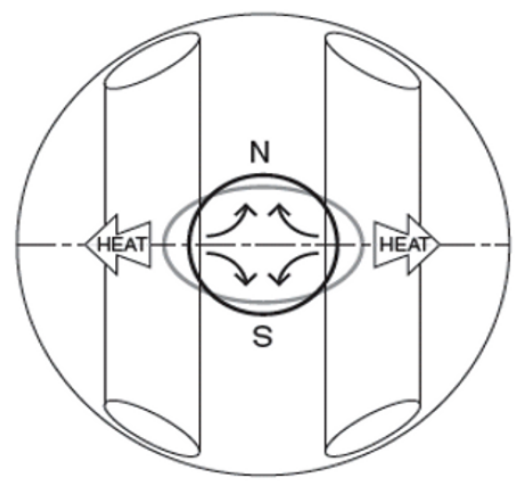

(b) Tp1

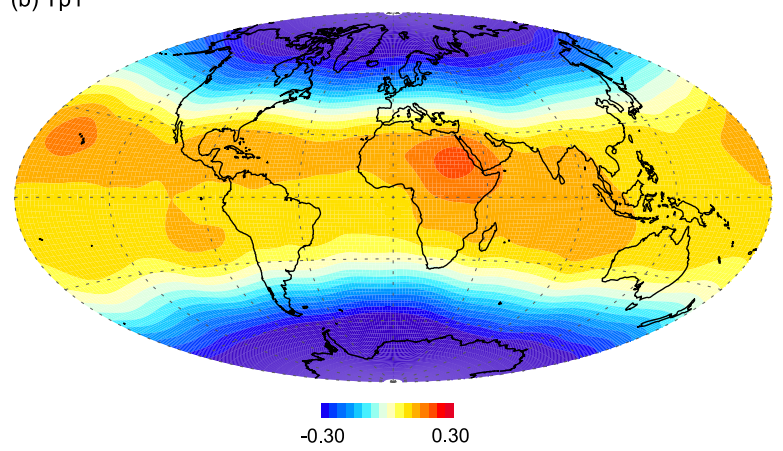

(d) Tp3

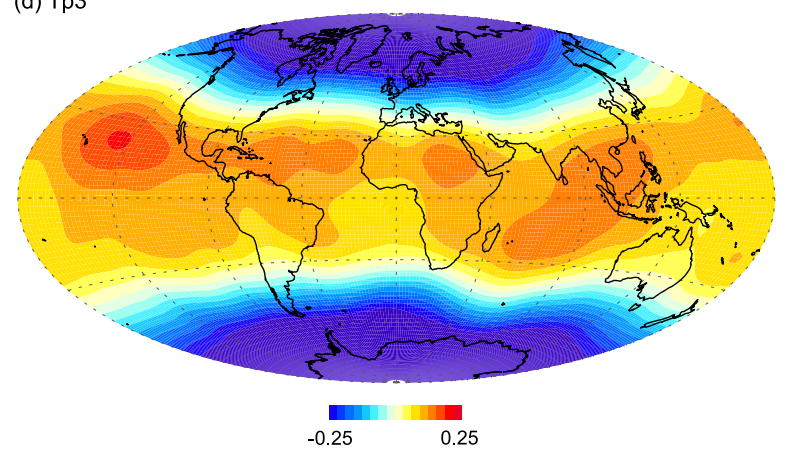

(f) $\mathrm{TH} 97$

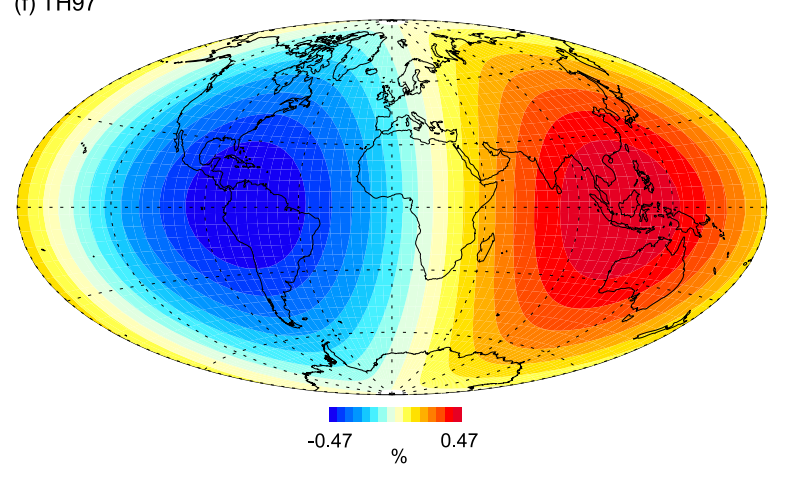

Fig. 5. Time-average inner-core boundary buoyancy flux anomalies normalized by the mean buoyancy flux: (a) Model T; (b) Model Tp1; (c) Model Tp2; (d) Model Tp3; (e) A schematic latitudinal model from Yoshida et al. (1996); (f) Seismic velocity anomalies from Tanaka and Hamaguchi (1997).

flux patterns among the various cases are quite remarkable (Figs. 3-5) considering the small variations between the imposed outer boundary patterns (Fig. 2). When the tanh function is strongly spread and the $\delta q$ vs. $\delta v_{s}$ relation is nearly linear, variations with respect to the purely thermal $\mathrm{T}$ case are expected to be modest (as in case Tp2). Post-Perovskite effects are therefore best seen when the transition is sharp (as in cases $\mathrm{Tp} 1$ and $\mathrm{Tp} 3$ ). Comparing cases $\mathrm{T}$ and $\mathrm{Tp} 2$ with cases Tp1 and Tp3, we find that accounting for postPerovskite often yields a westward shift in the locations of magnetic field, flow, and inner-core buoyancy flux structures. Perhaps more interesting, we detect a change in the large-scale symmetry properties of the steady dynamo from $\mathrm{T}$ and $\mathrm{Tp} 2$ to Tp1 and Tp3, from a mid-latitudes vortex that encompasses nearly the entire southern hemisphere to a vortex confined to the southern Atlantic only, and from two isolated positive inner boundary buoyancy flux structures to a more spread distribution.

\section{Discussion}

4.1 Geometry of the coupling between the outer and inner boundaries

The kinematic relation between the flow and the magnetic field is well-understood: Cyclones are associated with surface downwellings (Olson et al., 1999) that concentrate magnetic field to produce intense non-axisymmetric patches on the outer boundary (Olson and Christensen, 2002). This relation is evidenced in the good correlation between locations of intense magnetic field structures on the edge of the tangent cylinder (Fig. 3) and locations of cyclones (Fig. 4). However, depending on the imposed boundary pattern, different steady thermal winds are formed, resulting in different locations of these cyclones and their associated intense magnetic field patches.

Less straight-forward is the relation between the boundary-driven flow at the top of the shell and the buoyancy flux at the inner-core boundary. In rapidly rotating 

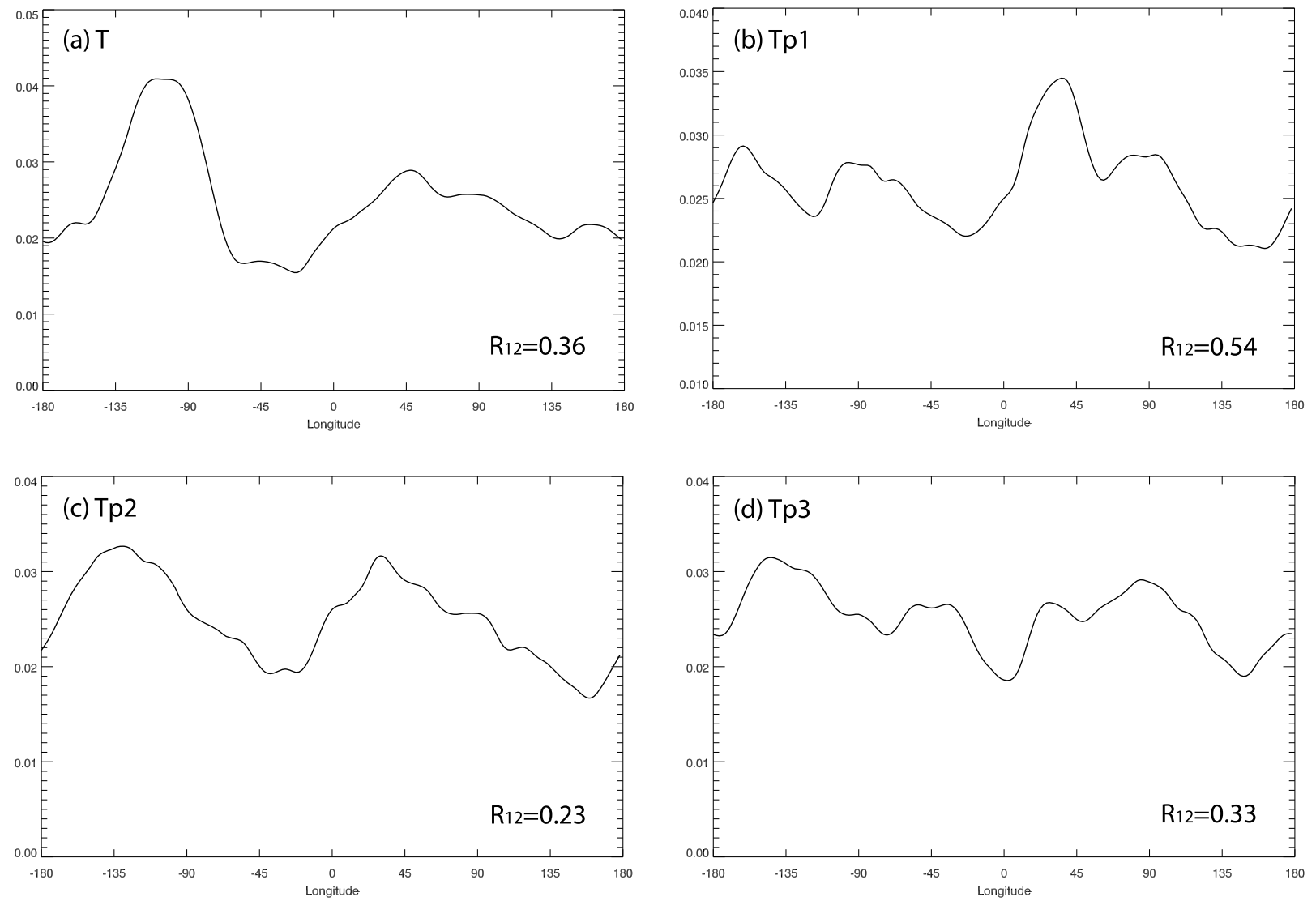

Fig. 6. Latitudinal averages of Fig. 5(a-d), where only positive values were considered. The ratios of the two lowest azimuthal modes $R_{12}$ are given for each case.

flows, nearly 2D strongly time-dependent $\hat{z}$-aligned Busse rolls that intersect the outer boundary at the edge of the tangent cylinder (Busse, 1975; Olson et al., 1999) are efficient in extracting buoyancy from the equatorial inner-core boundary at the longitude of the roll (Aubert et al., 2008). In order for such axial rolls to prevail on time-average, the thermal anomaly at the outer boundary should induce a thermal wind flow with very antisymmetric features with respect to the equator. Such a scenario is especially favorable when perfect equatorially-symmetric boundary conditions are imposed, e.g. $Y_{2}^{2}$ (Bloxham, 2002; Olson and Christensen, 2002; Aubert et al., 2007). When a multiharmonic mantle tomography model is used, the equatorial symmetry depends on the non-linear interaction between the homogeneous dynamo action and the boundary-driven flow as prescribed by the model parameters (Amit et al., 2008). In Aubert et al. (2008), the surface flow is antisymmetric with respect to the equator below Asia, and it is there that strong inner-core boundary buoyancy flux signal is present at the equatorial region.

If the resulting boundary-driven thermal wind flow deviates from perfect symmetry, the long-term time-average flow may get organized in quasi-axial inclined rolls. Such rolls must have small inclination with respect to the rotation axis to satisfy rotational constraints, but also the rolls should be tangent to the inner-core boundary in order to efficiently extract buoyancy. If the low-latitude edges of a pair of surface vortices appear at the same longitude but not the same latitude $\left(\phi_{0}, \theta_{n}\right)$ and $\left(\phi_{0}, \theta_{s}\right)$, the geometrical con- straint simply results in the deviation of both co-latitudes from the tangent cylinder $\theta_{i}$ by the amount of inclination $I$, i.e. $\theta_{n}=\theta_{i}-I$ and $\theta_{s}=\pi-\theta_{i}-I$, and the same applies for the inner-core boundary buoyancy flux deviation from the equator, i.e. $\theta_{q}=\pi / 2-I$ (see Appendix A.1 and Fig. 7). In this case one cyclone's low-latitude edge is inside the tangent cylinder.

The general case of both longitudinal and latitudinal displacements is more interesting (Appendix A.2). Assuming an arbitrary low-latitude edge of the southern vortex at $\left(0^{\circ} \mathrm{E}\right.$, $60^{\circ} \mathrm{S}$ ), we can obtain the longitudinal difference between the surface vortices $\delta$, the inclination of the roll with respect to the rotation axis $I$, and the spherical coordinates where the roll touches the inner-core boundary $\phi_{q}$ and $\theta_{q}$, all as functions of the co-latitude of the low-latitude edge of the northern vortex $\theta_{n}$. Figure 8 shows that, for example, if the northern vortex low-latitude edge is at $65^{\circ} \mathrm{N}$, then its longitude is $\delta \sim 82^{\circ} \mathrm{E}$, the roll is inclined by only $\sim 7^{\circ}$, and the roll touches the inner-core boundary at $\sim\left(37^{\circ} \mathrm{E}, 5^{\circ} \mathrm{N}\right)$. This illustrates that a roll with a modest inclination can connect two surface vortices seemingly distant in longitude while being tangent to the inner boundary.

Several more general points in Fig. 8 are worth-noting. First, when both surface vortices appear in the same latitude at the opposite hemispheres (in this case $\theta_{n}=30^{\circ}$ ), the roll touches the inner boundary at the equator $\left(\theta_{q}=90^{\circ}\right)$, as expected. If $\theta_{q}$ is smaller/larger than $90^{\circ}$ then the northern/southern vortex is at higher latitudes respectively, as in Fig. 7. Second, vortices closer to the tangent cylin- 


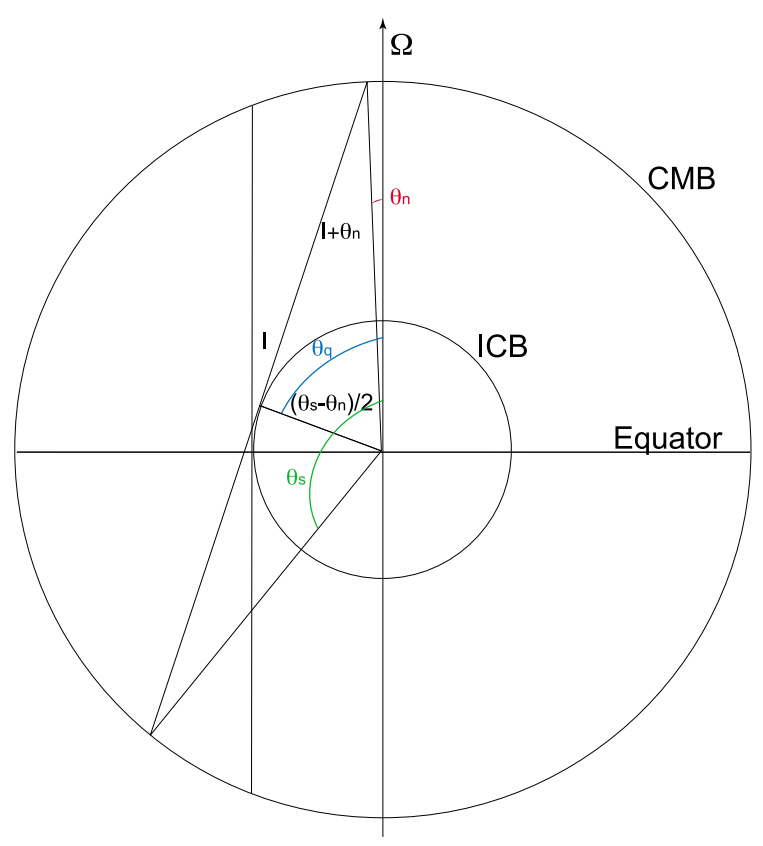

Fig. 7. Geometry of a latitudinally inclined roll tangent to the inner-core boundary.

der yield rolls with smaller inclinations. Finally, the ratio $\phi_{q} / \delta \sim 0.5$, i.e. the longitude where the roll touches the inner boundary is roughly the average longitude of the two surface vortices. The further apart the surface vortices (larger $\delta$ ), the larger the ratio (i.e. the roll touches the inner boundary closer to the lower latitude vortex), and the larger the inclination.

Quasi-axial convective rolls may provide an explanation for the locations of the positive anomalies in the timeaverage buoyancy flux. For example, in case Tp1 lowlatitude edges of cyclonic flows found at $\sim\left(45^{\circ} \mathrm{E}, 50^{\circ} \mathrm{S}\right)$ and $\sim\left(20^{\circ} \mathrm{E}, 65^{\circ} \mathrm{N}\right)$ produce a positive inner boundary buoyancy flux at $\sim\left(35^{\circ} \mathrm{E}, 15^{\circ} \mathrm{N}\right)$ (Fig. $\left.5(\mathrm{~b})\right)$. This is in agreement with our predictions that $\phi_{q}$ is roughly the average longitude of the two surface vortices, and $\theta_{q}$ leans to the northern hemisphere where the cyclonic flow is at higher latitudes. These two vortices can be identified in all four models (Fig. 4), and so does the resulting positive buoyancy flux anomaly in northern equatorial Africa (Fig. 5). Likewise, the central American buoyancy flux structures are generated by quasiaxial rolls linking the prominent cyclone below north America with a cyclone in the southern hemisphere at about longitude $180^{\circ}$.

We can try to estimate the inclination of the rolls in our models by considering intense magnetic flux patches. As an example, in Fig. 9 we show polar views of the timeaverage radial magnetic field on the outer boundary of case Tp3. The intense flux patches centered in $\left(186^{\circ} \mathrm{E}, 66^{\circ} \mathrm{N}\right)$ and $\left(171^{\circ} \mathrm{E}, 64^{\circ} \mathrm{S}\right)$ are concentrated by surface cyclones (Fig. 4(d)), and the quasi-axial roll connecting them generates the peak in positive buoyancy flux below the Pacific (Fig. 5(d)). Substituting these coordinates to (A.14), we find that the convective roll is inclined by only $2^{\circ}$ with respect to the rotation axis. We repeated these estimates for the other cases, and found that the inclinations are generally
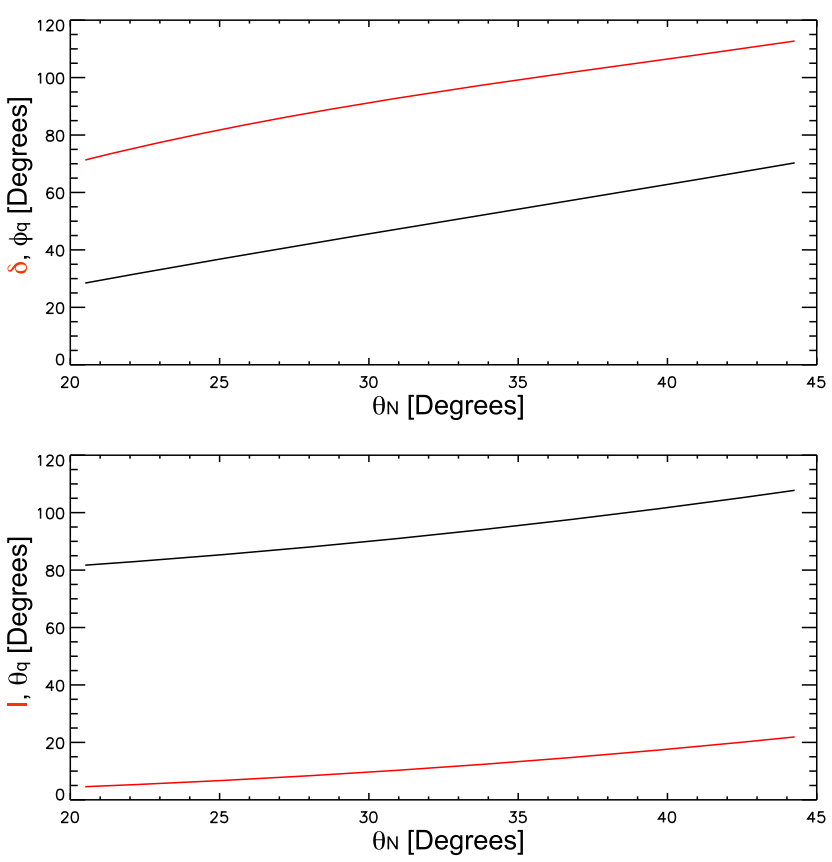

Fig. 8. A general inclined roll tangent to the inner-core boundary: Longitudinal difference between surface vortices (top, red); Longitude of point touching the inner-core boundary (top, black); Inclination (bottom, red); Latitude of point touching the inner-core boundary (bottom, black), all as functions of the co-latitude of the northern vortex (the southern vortex is fixed at $\left.\left(0^{\circ} \mathrm{E}, 60^{\circ} \mathrm{S}\right)\right)$. The left end side in both panels is the tangent cylinder.

larger (but still small), in the range $2^{\circ}-12^{\circ}$.

The positive low-latitude inner boundary buoyancy flux structures appear at the northern hemisphere for all cases (see also Aubert et al., 2008), suggesting that the southern hemisphere cyclonic flows reach lower latitudes than the northern ones. A possible reason for this difference is the large anticlockwise vortex in mid-latitudes of the southern Atlantic, which is common to all cases and does not have a northern counterpart. Although the net circulation of this vortex is anticyclonic, its limbs often exhibit local curvatures with negative radial vorticity (see e.g. eastward limb in Fig. 4(b) and westward limb in Fig. 4(d)). The associated local cyclonic motion at mid-latitudes of the southern hemisphere, as opposed to the generally weak flow at mid-latitudes of the northern hemisphere, may result in quasi-axial rolls touching the inner boundary in the northern Atlantic hemisphere. This hypothesis however does not explain the northward location of the positive low-latitude structures in the Pacific hemisphere, which could originate from a different physical process.

We stress that the concept of inclined convective rolls proposed here is relevant only for the long-term timeaverage part of the flow. The steady flow is driven by the mantle heterogeneity, which may contain some deviations from equatorial symmetry. The instantaneous flow, which is strongly affected by rapid rotation effects, is organized in nearly 2D axial Busse rolls.

\subsection{Comparison with observations}

Before comparing with observations, it is important to keep in mind that due to computational limitations the control parameters in numerical dynamo models are very far 
$180^{\circ}$

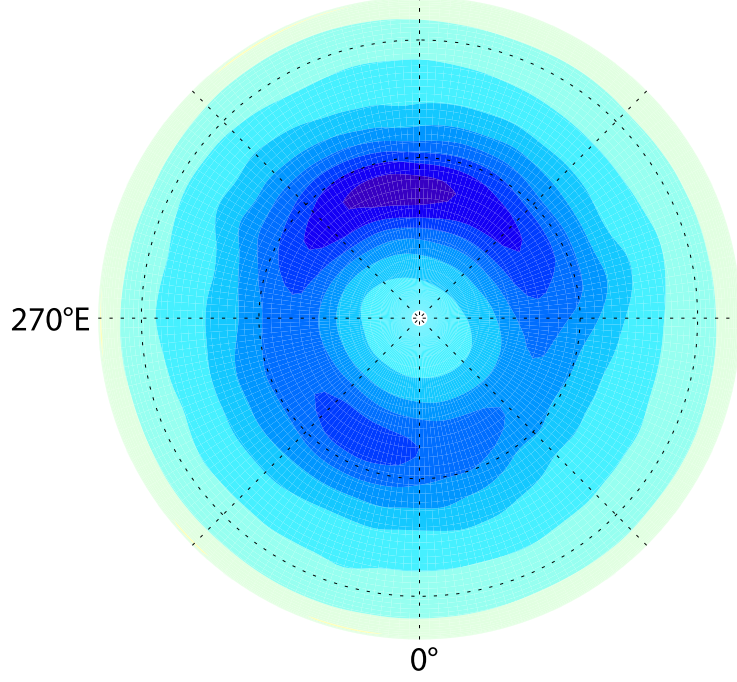

$0^{\circ}$

$90^{\circ} \mathrm{E} \quad 270^{\circ} \mathrm{E}$

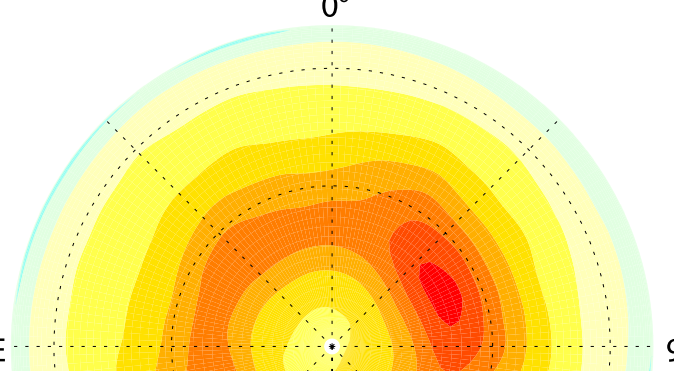

Fig. 9. North (left) and south (right) polar views of Fig. 3(d) (same scale).

from Earth-like conditions, and therefore the results of these simulations should be taken with caution. Especially, the Ekman number $E$ is far too large in the models. The models presented in this study contain an intermediate $E$ to allow for long time-averaging. Takahashi et al. (2008b) studied the effects of variable $E$ with a $Y_{2}^{2}$ heat flux pattern imposed on the outer boundary. They found that in their low $E$ models the direction of longitudinal shift of magnetic flux structures is opposite to the shift in the intermediate $E$ models of Olson and Christensen (2002), whereas the latitude of these patches is at the edge of the tangent cylinder for all $E$ values. They also noted that in their low $E$ models the magnetic Reynolds number $R m$ is lower. They speculated that the longitudinal shift may depend on $E, R m, \Lambda$ and $q^{*}$. It is worth noting that approaching Earth-like conditions in a subset of the control parameters might not necessarily yield more realistic models (for a detailed discussion see section 4 of Olson et al., 2009). For example, the Earth-like morphology of the instantaneous surface magnetic fields obtained at intermediate $E$ (e.g. Olson et al., 1999) is lost at low $E$ models which are too dipolar (Takahashi et al., 2008a)

Aubert et al. (2008) demonstrated that their dynamo model's time-average magnetic field, flow and inner boundary buoyancy flux patterns match rather well the timeaverage paleomagnetic field (Kelly and Gubbins, 1997), the historical time-average core flow just below the core-mantle boundary (Amit and Olson, 2006), and the seismic heterogeneity of the upper inner-core (Tanaka and Hamaguchi, 1997). They also showed the robustness of their results with two different volumetric buoyancy sources/sinks. However, Amit et al. (2008) illustrated that the time-average dynamo properties may be strongly-dependent on the model parameters. In this study our goal is not to match model output with geophysical observations. Instead, we examine dynamos with a fixed set of parameters, and compare the impact of various heat flux patterns on the time-average dynamo properties. These heat flux patterns may represent the combined effects of thermal and phase transition ori- gins of seismic lower mantle anomalies. Our philosophy is therefore primarily to detect changes associated with different boundary conditions, and then to observe whether the changes introduced by the inclusion of the post-Perovskite effect improve (or degrade) the recovery of observed patterns.

We consider the following observational (or observationally-inferred) geodynamo properties. The paleomagnetic field model averaged over the past five million years (Kelly and Gubbins, 1997) is given in Fig. 3(e) (from hereafter $K G 97$ ). Although several paleomagnetic field models were constructed using various methods and datasets, their non-axisymmetric features resemble (Constable, 2007). Figure 3(f) shows the time-average field for the period 1840-1990 based on the historical model gufml of Jackson et al. (2000). Two time-average core flow models of Amit and Christensen (2008) based on the inversions of the historical geomagnetic secular variation for the period 1840-1990 (Jackson et al., 2000) are shown in Fig. 4, one with the conventional frozen-flux approximation (Fig. 4(e), from hereafter $A C 08 F F$ ), the other includes a model of magnetic diffusion inferred from numerical dynamos (Fig. 4(f), from hereafter AC08DIF). These models were obtained with a purely helical flow assumption by solving a set of two coupled differential equations for the two flow potentials using a local central finite-difference numerical method on a regular grid (Amit and Olson, 2004). Amit and Christensen (2008) compared their frozen-flux and diffusive core flow models in sampled snapshots. They found that the large-scale circulation is similar, but the magnitude of the diffusive core flow is larger and some significant local differences appear. This is also apparent for the time-averages shown in Fig. 4(e-f). Both time-average flow models are governed by large anticlockwise vortices below the southern Atlantic and below Asia with a broad westward jet at mid-latitudes of the southern hemisphere. We denote two significant local differences between the two models: (1) The cyclone below Asia in AC08DIF is stronger, and (2) the anticyclone below 
North America in $A C 08 F F$ splits into an anticyclone below the North Atlantic and a cyclone below North America in AC08DIF. Finally, we illustrate the robust observation of latitudinal inner-core seismic anisotropy with pole-to-pole seismic velocities $\sim 3 \%$ faster that in the equatorial plane (Morelli et al., 1986; Creager, 1992; Song and Helmberger, 1993) by a conceptual model of isostatic adjustment due to Busse rolls (from Yoshida et al. (1996), Fig. 5(e), from hereafter YSK96). We also show the observed longitudinal seismic heterogeneity of the upper inner core (Tanaka and Hamaguchi (1997), Fig. 5(f), from hereafter TH97). Several studies proposed more detailed upper inner-core longitudinal heterogeneity (e.g. Niu and Wen, 2001), however the most robust feature in these models is the east-west hemispherical variation.

Comparing these observed steady geodynamo features with the time-average tomographic dynamo models' output (Figs. 3-5), we find some gross common features. These include the dominance of the axial dipole and some highlatitude intense patches in the radial magnetic field, the large anti-clockwise vortex below the southern Atlantic including westward flow at mid-latitudes of the southern Atlantic hemisphere associated with the well-known observed westward drifting magnetic field features there (Jackson et al., 2000), and the dominant $Y_{2}^{0}$ mode in the inner boundary buoyancy flux. However, we wish to make a more detailed comparison, especially the longitudinal variations among the different models and the observations.

Our results show some improvement in recovery of important observational features when accounting for postPerovskite. In the purely thermal case $\mathrm{T}$ and in the weakly non-linear case Tp2 an anticlockwise vortex extends throughout the entire southern hemisphere (Figs. 4(a) and 4(c)). In contrast, in the strongly non-linear cases Tp1 and Tp3 this vortex is confined to the southern Atlantic (Figs. 4(b) and 4(d)), in agreement with the Atlantic/Pacific hemispherical dichotomy in the geomagnetic secular variation (Christensen and Olson, 2003; Gubbins and Gibbons, 2004) as seen in the southern Atlantic anticlockwise vortex in $A C 08 F F$ (Fig. 4(e)) and more pronouncedly in ACO8DIF (Fig. 4(f)). In cases T and Tp2 the inner boundary buoyancy flux at low-latitudes contain two isolated positive anomalies below central America and east Africa (Figs. 5(a) and 5(c)), whereas in cases $\mathrm{Tp} 1$ and $\mathrm{Tp} 3$ the distribution is more spread with larger relative $m=1$ signal in case Tp1 (Figs. 5(b) and 5(d)), possibly suggestive of the dominant $m=1$ azimuthal mode in TH97 (Fig. 5(f)).

Previous studies argued that the amount of longitudinal shift in the locations of surface field and flow structures depends on the Ekman (Olson and Christensen, 2002) or Peclet (Aubert et al., 2007) numbers. The paleomagnetic field model KG97 contains two northern hemisphere nonaxisymmetric structures below Canada and Siberia, and one southern hemisphere structure below $\sim 0^{\circ}$ longitude (Fig. 3(e)). Case T contains two northern hemisphere structures in very similar locations, but has two structures in the southern hemisphere (Fig. 3(a)). In contrast, in case Tp1 the two northern hemisphere patches are shifted westward, but the southern hemisphere contains one structure (Fig. 3(b)). Cases Tp2 and Tp3 also exhibit two intense northern patches and only one dominant southern patch (Figs. 3(c) and 3(d)). We argue that the spectral content of the radial magnetic field of cases Tp1, Tp2 and Tp3 agrees better with the paleomagnetic observation than case $\mathrm{T}$, while the successful recovery of the correct longitudes in the northern hemisphere of case $\mathrm{T}$ is likely fortuitous. The time-average field from the historical model gufml contains two intense structures at each hemisphere. Although the reliability of this model is much higher, it spans a much shorter time interval, and therefore it is probable that this model contains transient features.

\section{Summary}

If non-axisymmetric boundary conditions are imposed on the dynamo by the heterogeneous lower mantle, the dynamo reaches a dynamical compromise between rotational constraints which favor axial rolls (Busse, 1975) and boundarydriven thermal wind flows that may include some equatorial asymmetry (Amit et al., 2008). The resulting convective quasi-axial rolls extract more buoyancy from lowlatitudes. The rolls connect opposite hemisphere surface cyclones somewhat apart in longitude, and produce positive buoyancy structures at low-latitudes of the inner boundary slightly off the equator. Our models predict that this offset leans toward the northern hemisphere. We hypothesize that the large anticlockwise vortex below the southern Atlantic, a robust feature in inverted core flows from geomagnetic secular variation (Jackson, 1997; Hulot et al., 2002; Amit and Olson, 2006) is responsible for some of this northward offset. Cyclonic flows at the limbs of this vortex connect with higher-latitude northern hemisphere cyclones to form quasi-axial northerly-inclined convective rolls that touch the inner-core boundary at the northern Atlantic lowlatitudes.

Our results may shed light on the symmetry properties that the heterogeneous lower mantle induces on the geodynamo. The two non-axisymmetric high-latitude intense radial paleomagnetic field patches in the northern hemisphere of $K G 97$ are maintained by convergence associated with two cyclones in the northern hemisphere, as the ones in the time-average flow model ACO8DIF at similar longitudes (below North America and Asia). In contrast, the southern hemisphere displays one paleomagnetic field intense patch, indicating a different spectral content to the mantle control there. Extracting buoyancy from the inner-core boundary requires two vortices, one in each hemisphere, connected by a convective roll. Having two vortices in one hemisphere and one vortex in another allows for only one convective roll and thus one dominant positive buoyancy flux anomaly in the inner-core boundary. These symmetry properties seem to be better captured in the cases that account for post-Perovskite than in the purely thermal interpretation of the tomographic model.

We conclude that the relation between seismic and heat flux anomalies that accounts for post-Perovskite phase transitions as suggested by Nakagawa and Tackley (2008) may improve the consistency of the dynamo models with the geodynamo observations. A natural next step would be to incorporate chemical contributions to seismic shear velocity anomalies and to examine the consequences for the time- 
average geodynamo properties. Overall, future progress in the study of the geodynamical properties of the $\mathrm{D}^{\prime \prime}$ layer, better seismic tomography models of the lower mantle and the upper inner-core, more accurate geomagnetic and paleomagnetic field models, improved techniques of core flow inversions from geomagnetic secular variation, and better understanding of inner-core solidification mechanisms, will further promote the study of mantle control on the geodynamo and the coupling between the lower mantle and the upper inner-core by outer core convection.

Acknowledgments. Numerical calculations were performed at the Service de Calcul Parallelle, IPGP. We thank two anonymous reviewers for their useful comments that significantly improved the manuscript.

\section{Appendix A. Inclined Rolls}

\section{A.1 Latitudinal inclination}

Let $\theta_{i} \simeq 20.5^{\circ}$ be the co-latitude at which the tangent cylinder intercepts the core-mantle boundary, and consider two surface vortices at the same longitude but at different latitudes $\left(\phi_{0}, \theta_{n}\right)$ and $\left(\phi_{0}, \theta_{s}\right)$. These coordinates represent either very thin vortex filaments in a snapshot, or the lowlatitude edges of large-scale time-average vortices. The vortices are linked through the volume of the outer core by a roll with an inclination $I$ with respect to the axis of rotation and is tangent to the inner-core boundary (Fig. 7). The relations between $\theta_{n}, \theta_{s}$ and $I$ are:

$$
\begin{gathered}
\sin \left(I+\theta_{n}\right)=\frac{R_{\mathrm{i}}}{R_{\mathrm{o}}}=\sin \left(\theta_{i}\right) \\
I+\theta_{n}+\frac{\theta_{s}-\theta_{n}}{2}=\frac{\pi}{2}
\end{gathered}
$$

where $R_{\mathrm{i}}$ and $R_{\mathrm{o}}$ are the radii of the inner and outer cores. From (A.1) and (A.2) it is clear that the northern hemisphere vortex co-latitude is $\theta_{n}=\theta_{i}-I$ and the deviation of the southern hemisphere vortex from the tangent cylinder is $\pi-\theta_{s}=\theta_{i}+I$. Also, the co-latitude where the roll touches the inner-core boundary $\theta_{q}$ is

$$
\theta_{q}=\theta_{n}+\frac{\theta_{s}-\theta_{n}}{2}=\pi / 2-I
$$

In summary, the inclination of the roll by $I$ results in the same change in $\theta_{n}, \theta_{s}$ and $\theta_{q}$.

\section{A.2 General inclination}

Now consider two generic vortices just below the outer boundary $\left(r=R_{\mathrm{o}}\right)$ at $\left(0, \theta_{s}\right)$ and $\left(\delta, \theta_{n}\right)$. Once again the coordinates represent either very thin vortex filaments in a snapshot, or the low-latitude edges of large-scale time-average vortices. In a 3D Cartesian coordinate system $x y z$ with $z$ pointing to the rotation axis, their locations are $\left(R_{\mathrm{o}} \sin \theta_{s}, 0, R_{\mathrm{o}} \cos \theta_{s}\right)$ and $\left(R_{\mathrm{o}} \sin \theta_{n} \cos \delta, R_{\mathrm{o}} \sin \theta_{n} \sin \delta, R_{\mathrm{o}} \cos \theta_{n}\right)$. The vortices are again linked through the volume of the outer core by a roll that is tangent to the inner-core boundary. Using simple linear algebra, we can represent the roll by a line and the inner-core boundary by a sphere and look for a single solution. The general equation for a line is

$$
\frac{x-x_{0}}{a}=\frac{y-y_{0}}{b}=\frac{z-z_{0}}{c}
$$

Substituting the coordinates of the two points, we get

$$
\begin{aligned}
& \frac{\sin \theta_{n} \sin \delta}{\sin \theta_{n} \cos \delta-\sin \theta_{s}}\left(x-R_{\mathrm{o}} \sin \theta_{s}\right) \\
& \quad=y=\frac{\sin \theta_{n} \sin \delta}{\cos \theta_{n}-\cos \theta_{s}}\left(z-R_{\mathrm{o}} \cos \theta_{s}\right)
\end{aligned}
$$

The equation of the inner-core boundary surface $\left(r=R_{\mathrm{i}}=\right.$ $\left.0.35 R_{\mathrm{o}}\right)$ is

$$
x^{2}+y^{2}+z^{2}=R_{\mathrm{i}}^{2}
$$

We can re-arrange (A.5) to write $x$ and $z$ as functions of $y$, and substitute into (A.6) to get a quadratic equation for $y$ :

$$
\begin{array}{r}
\frac{1-\sin \theta_{n} \sin \theta_{s} \cos \delta-\cos \theta_{n} \cos \theta_{s}}{\sin ^{2} \theta_{n} \sin ^{2} \delta} y^{2} \\
-\frac{1-\sin \theta_{n} \sin \theta_{s} \cos \delta-\cos \theta_{n} \cos \theta_{s}}{\sin \theta_{n} \sin \delta} R_{\mathrm{o}} y \\
+\frac{R_{\mathrm{o}}^{2}-R_{\mathrm{i}}^{2}}{2}=0
\end{array}
$$

Rolls that do not touch the inner-core boundary correspond to zero solutions for (A.7), while two solutions represent non-existing rolls that are blocked by the inner-core. We wish to find the rolls tangent to the inner-core boundary, i.e. one solution to (A.7). Setting the discriminant to zero, we obtain a constraint for the vortices coordinates

$$
\cos \delta=\frac{2\left(R_{\mathrm{i}} / R_{\mathrm{o}}\right)^{2}-1-\cos \theta_{n} \cos \theta_{s}}{\sin \theta_{n} \sin \theta_{s}}
$$

and the single solution is

$$
y=\frac{\sin \theta_{n} \sin \delta}{2} R_{\mathrm{o}}
$$

Using (A.5) we can obtain the single solution for $x$ and $z$

$$
\begin{aligned}
& x=\frac{\sin \theta_{n} \cos \delta+\sin \theta_{s}}{2} R_{\mathrm{o}} \\
& z=\frac{\cos \theta_{n}+\cos \theta_{s}}{2} R_{\mathrm{o}}
\end{aligned}
$$

Note that of the four spherical coordinates (two for each vortex) there are only two free parameters, because one longitude is arbitrary $\left(\phi_{s}=0\right)$, and due to the additional constraint (A.8). Using (A.9)-(A.11), the spherical coordinates of the point where the roll touches the inner-core boundary are given by

$$
\begin{aligned}
& \phi_{q}=\tan ^{-1}\left(\frac{\sin \theta_{n} \sin \delta}{\sin \theta_{n} \cos \delta+\sin \theta_{s}}\right) \\
& \theta_{q}=\tan ^{-1}\left(\frac{\sqrt{\sin ^{2} \theta_{n}+\sin ^{2} \theta_{s}+2 \sin \theta_{n} \sin \theta_{s} \cos \delta}}{\cos \theta_{n}+\cos \theta_{s}}\right)
\end{aligned}
$$

and the inclination of the roll with respect to the rotation axis is

$$
I=\tan ^{-1}\left(\frac{\sqrt{\sin ^{2} \theta_{n}+\sin ^{2} \theta_{s}-2 \sin \theta_{n} \sin \theta_{s} \cos \delta}}{\left(\cos \theta_{n}-\cos \theta_{s}\right)^{2}}\right)
$$


If both surface vortices are on the edge of the tangent cylinder, i.e. $\theta_{n}=\theta_{s}=\theta_{i}$, we obtain the trivial solution $\delta=0$ and the roll is not inclined with respect to the rotation axis. To make progress, we arbitrarily set $\theta_{s}=150^{\circ}\left(60^{\circ} \mathrm{S}\right)$ as the low-latitude edge of the southern vortex, leaving $\delta, \phi_{q}, \theta_{q}$ and $I$ as functions of the co-latitude $\theta_{n}$ only. Solutions are shown in Fig. 8.

\section{References}

Amit, H. and U. Christensen, Accounting for magnetic diffusion in core flow inversions from geomagnetic secular variation, Geophys. J. Int., 175, 913-924, 2008.

Amit, H. and P. Olson, Helical core flow from geomagnetic secular variation, Phys. Earth Planet. Inter., 147, 1-25, 2004.

Amit, H. and P. Olson, Time-average and time-dependent parts of core flow, Phys. Earth Planet. Inter., 155, 120-139, 2006.

Amit, H., J. Aubert, G. Hulot, and P. Olson, A simple model for mantledriven flow at the top of Earth's core, Earth Planets Space, 60, 845-854, 2008.

Aubert, J., H. Amit, and G. Hulot, Detecting thermal boundary control in surface flows from numerical dynamos, Phys. Earth Planet. Inter., 160, 143-156, 2007.

Aubert, J., H. Amit, G. Hulot, and P. Olson, Thermo-chemical flows couple Earth's inner core growth to mantle heterogeneity, Nature, 454, 758$761,2008$.

Bloxham, J., Time-independent and time-dependent behaviour of highlatitude flux bundles at the core-mantle boundary, Geophys. Res. Lett., 29, doi:10.1029/2001g1014543, 2002.

Bloxham, J. and D. Gubbins, Thermal core-mantle interactions, Nature, 325, 511-513, 1987.

Busse, F., A model of the geodynamo, Geophys. J. R. Astron. Soc., 42, 437-459, 1975.

Carlut, J. and V. Courtillot, How complex is the time-averaged geomagnetic field over the last 5 million years?, Geophys. J. Int., 134, 527-544, 1998.

Christensen, U. and P. Olson, Secular variation in numerical geodynamo models with lateral variations of boundary heat flow, Phys. Earth Planet. Inter., 138, 39-54, 2003.

Constable, C., Non-dipole field, in Encyclopedia of Geomagnetism and Paleomagnetism, edited by Gubbins, D. and E. Herrera-Bervera, 1054 pp, Springer, The Netherlands, 2007.

Creager, K., Anisotropy of the inner core from differential travel-times of the phases PKP and PKIKP, Nature, 356, 309-314, 1992.

Deschamps, F., J. Trampert, and P. Tackley, Thermo-chemical structure of the lower mantle: seismological evidence and consequences for geodynamics, in Superplume: Beyond Plate Tectonics, edited by Yuen, D., S. Maruyama, S. Karato, and B. Windely, 568 pp, Springer, The Netherlands, 2007.

Glatzmaier, G., R. Coe, L. Hongre, and P. Roberts, The role of the Earth's mantle in controlling the frequency of geomagnetic reversals, Nature, 401, 885-890, 1999.

Gubbins, D. and S. Gibbons, Low Pacific secular variation, in Timescales of the paleomagnetic field, edited by Channell, J., D. Kent, W. Lowrie, and J. Meert, 320 pp, Geophysical monograph series Vol. 145, Washington D.C., 2004

Gubbins, D., A. Willis, and B. Sreenivasan, Correlation of Earth's magnetic field with lower mantle thermal and seismic structure, Phys. Earth Planet. Inter., 162, 256-260, 2007.

Holme, R., Large-scale flow in the core, in Treatise on Geophysics Vol. 8 , edited by Olson, P., 376 pp, Elsevier Science, London, 2007.

Hulot, G., C. Eymin, B. Langlais, M. Mandea, and N. Olsen, Small-scale structure of the geodynamo inferred from Oersted and Magsat satellite data, Nature, 416, 620-623, 2002.

Iitaka, T., K. Hirose, K. Kawamura, and M. Murakami, The elasticity of the $\mathrm{MgSiO} 3$ post-Perovskite phase in the Earth's lowermost mantle, Nature, 430, 442-445, 2004.

Jackson, A., Time-dependency of tangentially geostrophic core surface motions, Phys. Earth Planet. Inter., 103, 293-311, 1997.

Jackson, A., A. Jonkers, and M. Walker, Four centuries of geomagnetic secular variation from historical records, Phil. Trans. R. Soc. Lond., A358, 957-990, 2000.

Kelly, P. and D. Gubbins, The geomagnetic field over the past 5 million years, Geophys. J. Int., 128, 315-330, 1997.
Labrosse, S., J.-W. Hernlund, and N. Coltice, A crystallizing dense magma ocean at the base of the Earth's mantle, Nature, 450, 866-869, 2007.

Lay, T., E.-J. Garnero, and Q. Williams, Partial melting in a thermochemical boundary layer at the base of the mantle, Phys. Earth Planet. Inter., 146, 441-467, 2004.

Lay, T., J. Hernlund, E. Garnero, and M. Thorne, A post-Perovskite lens and $\mathrm{D}^{\prime \prime}$ heat flux beneath the central Pacific, Science, 314, 1272-1276, 2006.

Le Bars, M. and A. Davaille, Whole layer convection in a heterogeneous planetary mantle, J. Geophys. Res., 109, Bo3403, 2004.

Masters, G., S. Johnson, G. Laske, and H. Bolton, A shear-velocity model of the mantle, Philos. Trans. R. Soc. Lond. Ser., A354, 1385-1411, 1996.

Masters, G., G. Laske, H. Bolton, and A. Dziewonski, The relative behavior of shear velocity, bulk sound velocity, and compressional velocity in the mantle: Implications for chemical and thermal structure, in Earth's deep interior, edited by Karato, S., A. Forte, R. Liebermann, G. Masters, and L. Stixrude, 297 pp, AGU monograph Vol. 117, Washington D.C., 2000 .

Matsumoto, N., A. Namiki, and I. Sumita, influence of a basal thermal anomaly on mantle convection, Phys. Earth Planet. Inter., 157, 208222, 2006.

Morelli, A., A. Dziewonski, and J. Woodhouse, Anisotropy of the inner core inferred from PKIKP travel-times, Geophys. Res. Lett., 13, 1545$1548,1986$.

Murakami, M., K. Hirose, N. Sata, Y. Ohishi, and K. Kawamura, PostPerovskite phase transition in MgSiO3, Science, 304, 855-858, 2004.

Murakami, M., S. Sinogeikin, J. Bass, and J. Li, Sound velocity of $\mathrm{MgSiO} 3$ Perovskite to mbar pressure, Earth Planet. Sci. Lett., 256, 47-54, 2007.

Nakagawa, T. and P. Tackley, Lateral variations in CMB heat flux and deep mantle seismic velocity caused by a thermal-chemical-phase boundary layer in 3D spherical convection, Earth Planet. Sci. Lett., 271, 348-358, 2008.

Ni, S., E. Tan, M. Gurnis, and D. Helmberger, Sharp sides to the African super plume, Science, 296, 1850-1852, 2002.

Niu, F. and L. Wen, Hemispherical variations in seismic velocity at the top of the Earth's inner core, Nature, 410, 1081-1084, 2001.

Oganov, A. and S. Ono, Theoretical and experimental evidence for a postPerovskite phase of MgSiO3 in Earth's D" layer, Nature, 430, 445-448, 2004.

Olson, P. and U. Christensen, The time averaged magnetic field in numerical dynamos with nonuniform boundary heat flow, Geophys. J. Int., 151, 809-823, 2002.

Olson, P., U. Christensen, and G. Glatzmaier, Numerical modeling of the geodynamo: Mechanisms of field generation and equilibration, $J$. Geophys. Res., 104, 10383-10404, 1999.

Olson, P., P. Driscoll, and H. Amit, Dipole collapse and reversal precursors in a numerical dynamo, Phys. Earth Planet. Inter., 173, 121-140, 2009.

Ritsema, J., A. McNamara, and A. Bull, Tomographics filtering of geodynamic models: implications for model interpretation and large-scale mantle structure, J. Geophys. Res., 112, doi:10.1029/2005GL023887, 2007.

Song, X. and D. Helmberger, Anisotropy of Earth's inner-core, Geophys. Res. Lett., 20, 2591-2594, 1993.

Stackhouse, S., J. Brodholt, and G. Price, Elastic anisotropy of FeSiO3 end-member of the Perovskite and post-Perovskite phase, Geophys. Res. Lett., 33, L01304, doi:10.1029/2005GL023887, 2006.

Su, W.-J. and A. Dziewonski, Simultaneous inversion for 3-D variations in shear and bulk velocity in the mantle, Phys. Earth Planet. Inter, 100, 135-156, 1997.

Su, W.-J., R. Woodward, and A. Dziewonski, Degree-12 model of shear velocity heterogeneity in the mantle, J. Geophys. Res., 99, 6945-6980, 1994.

Tackley, P., Strong heterogeneity caused by deep mantle layering, Geochem. Geophys. Geosyst., 3, 1024, 2002.

Takahashi, F., M. Matsushima, and Y. Honkura, Scale variability in convection-driven MHD dynamos at low Ekman number, Phys. Earth Planet. Inter., 167, 168-178, 2008a.

Takahashi, F., H. Tsunakawa, M. Matsushima, N. Mochizuki, and Y. Honkura, Effects of thermally heterogeneous structure in the lowermost mantle on the geomagnetic field strength, Earth Planet. Sci. Lett., 272, 738-746, 2008b.

Tanaka, S. and H. Hamaguchi, Degree one heterogeneity and hemispherical variation of anisotropy in the inner core from PKP(BC)-PKP(DF) times, J. Geophys. Res., 102, 2925-2938, 1997.

Thurber, C. and J. Ritsema, Theory and observations-seismic tomography and inverse methods, in Treatise on Geophysics. Vol. 1, edited by 
Romanowicz, B. and A. Dziewonski, 872 pp, Elsevier Science, London, 2007.

To, A., B. Romanowicz, Y. Capdeville, and N. Takeuchi, 3D effects of sharp boundaries at the borders of the African and Pacific superplumes: observation and modeling, Earth Planet. Sci. Lett., 233, 137-153, 2005.

Tsuchiya, T. and J. Tsuchiya, Effect of impurity on the elasticity of Perovskite and post-Perovskite: Velocity contrast across the postPerovskite transition in $(\mathrm{Mg}, \mathrm{Fe}, \mathrm{Al})(\mathrm{Si}, \mathrm{Al}) \mathrm{O} 3$, Geophys. Res. Lett., 33, L12S04, 2006.

Wentzcovitch, R., T. Tsuchiya, and J. Tsuchiya, MgSiO3 post-Perovskite at $\mathrm{D}^{\prime \prime}$ conditions, Proc. Nat. Acad. Sci., 103, 543-546, 2006.

Wicht, J., Inner-core conductivity in numerical dynamo simulations, Phys. Earth Planet. Inter., 132, 281-302, 2002.

Williams, Q. and E.-J. Garnero, Seismic evidence for partial melt at the base of Earth's mantle, Science, 273, 1528-1530, 1998.
Williams, Q., J. Revenaugh, and E.-J. Garnero, A correlation between ultra-low basal velocities in the mantle and hot spots, Science, 281, 546549, 1996.

Willis, A., B. Sreenivasan, and D. Gubbins, Thermal core-mantle interaction: exploring regimes for locked dynamo action, Phys. Earth Planet Inter., 165, 83-92, 2007.

Wookey, J., S. Stackhouse, J.-M. Kendall, J. Brodholt, and G. Price, Efficacy of the post-Perovskite phase as an explanation for lowermost mantle seismic properties, Nature, 438, 1004-1007, 2005.

Yoshida, S., I. Sumita, and M. Kumazawa, Growth model of the inner core coupled with the outer core dynamics and the resulting elastic anisotropy, J. Geophys. Res., 101, 28085-28103, 1996.

H. Amit (e-mail: Hagay.Amit@univ-nantes.fr) and G. Choblet 